\title{
Asma grave en pediatría: resultados de la implementación de un protocolo especial de atención
}

\author{
Severe asthma in pediatrics: Outcomes of the implementation of \\ a special health care protocol
}

\author{
Dra. Verónica Giubergia ${ }^{a}$ Dra. María J. Ramirez Farías ${ }^{a}$ Dra. Verónica Péreza, \\ Lic. Adriana González $z^{b}$ Lic. Nancy Crespi ${ }^{a}$, Lic. Nora Fridman ${ }^{c}$ y Dr. Claudio Castaños ${ }^{a}$
}

\section{RESUMEN}

Introducción. El asma grave representa 5-7\% del total de asmáticos. La OMS propuso un protocolo de seguimiento para categorizarlos como asma grave resistente al tratamiento (AGRT) o asma grave de difícil control (AGDC).

Objetivo. Analizar las características clínicas, funcionales y terapéuticas de pacientes con AGRT o AGDC.

Métodos. Estudio transversal, observacional y analítico para evaluar el diagnóstico, grado de control (clínico y funcional), comorbilidades, adherencia al tratamiento, técnica inhalatoria y factores ambientales en pacientes con asma grave. Resultados. Se incluyeron 69 pacientes: AGRT $(\mathrm{n}=33)$ y AGDC $(\mathrm{n}=36)$. El $100 \%$ del grupo con AGRT fue hospitalizado previamente por asma vs. 87,8\% del grupo con AGDC $(p=0,03)$. El 63\% del grupo AGRT requirió cuidados intensivos (UCI)), 82\%, asistencia ventilatoria y uno fue traqueostomizado. En el AGDC, 54\% requirió internación en la UCI, y $33 \%$, asistencia ventilatoria $(\mathrm{p}=0,03)$.

La espirometría basal fue normal en el AGDC; se observó incapacidad ventilatoria obstructiva leve en el AGRT $(p=0,009)$.

En el AGDC, hubo menor cumplimiento del tratamiento $(p=0,01)$. Se requirieron dosis mayores de corticoides inhalados en AGRT $(p=0,0001)$. Omalizumab fue indicado en AGRT $(p=0,0001)$. A los 6 meses de seguimiento, más del 75\% de los niños en ambos grupos presentó asma controlada.

Conclusiones. Se observó significativa falta de adherencia al tratamiento en el grupo AGDC. Se redujeron las dosis de tratamiento en este grupo. Se logró controlar la enfermedad en un alto porcentaje de niños con AGRT y AGDC. Palabras clave: asma grave, resistencia al tratamiento, prevención y control, programa.

http:/ / dx.doi.org/10.5546/aap.2018.105

Texto completo en inglés:

http:/ / dx.doi.org/10.5546/ aap.2018.eng.105.

Financiamiento:

Ninguno que declarar.

Conflicto de intereses:

Ninguno que declarar.

Recibido: 21-4-2017

Aceptado: 5-9-2017
Cómo citar: Giubergia V, Ramirez Farías MJ, Pérez V, et al. Asma grave en pediatría: resultados de la implementación de un protocolo especial de atención. Arch Argent Pediatr 2018;116(2):105-111.

\section{GLOSARIO}

AG: Asma grave

AGDC: Asma grave de difícil control AGRT: Asma grave resistente al tratamiento

ARM: Asistencia respiratoria mecánica

AV: Asistencia ventilatoria

CI: Corticoides inhalados

CVF: Capacidad vital forzada

ERS/ATS: European Respiratory

Society/American Thoracic Society

FEF: Flujo espiratorio forzado al

$25 / 75 \%$ de la capacidad vital forzada

LABA: Broncodilatadores de acción

prolongada

OMS: Organización Mundial de la

Salud

PANAG: Programa de Atención de

Niños con Asma Grave Problemática

TACAR: Tomografía axial computada de alta resolución

TCA: Test de control del asma

UCI: Unidad de Cuidados Intensivos

$\mathrm{VEF}_{1}$ :Volumen espiratorio forzado en el primer segundo

$\mathrm{VEF}_{1} / \mathrm{CVF}$ : Relación entre el volumen espiratorio forzado en el primer segundo y la capacidad vital forzada.

\section{INTRODUCCIÓN}

El asma es la enfermedad crónica más frecuente en la infancia y la principal causa de morbilidad pediátrica. ${ }^{1}$ Existen, aproximadamente, 300000000 de personas con asma, con la mayor prevalencia en edad pediátrica. La Argentina se ubica en un rango intermedio: $16,4 \%$ en niños de 6-7 años y $10,9 \%$ en los de $13-14 .^{1,2}$ 
En nuestro país, el asma ocasiona más de 400 muertes ( $10 \%$ en pacientes de 5 a 39 años) y más de 15000 hospitalizaciones anuales. ${ }^{1,3}$

Esto ocurre en individuos con asma más grave, especialmente, en los casos no controlados. El asma grave (AG) representa el 5-7\% del total de asmáticos. Constituyen un grupo pequeño, muy vulnerable. ${ }^{1,4,5}$ Presentan crisis y hospitalizaciones frecuentes y se encuentran en riesgo de desarrollar efectos adversos debido a las altas dosis de tratamiento con corticoides. Estos niños utilizan 15 veces más los servicios de emergencias y se internan 20 veces más que aquellos con asma leve o moderada. ${ }^{6}$

En los últimos años, la Organización Mundial de la Salud (OMS) propuso un protocolo para el seguimiento de pacientes con AG no controlada que evaluaba distintos factores que aportaban morbilidad, lo que facilitó su categorización y tratamiento. Este abordaje permitió clasificar el asma como AG de difícil control (AGDC) por causas modificables, con buena evolución clínica, o como AG resistente al tratamiento (AGRT), cuya evolución y tratamiento pueden ser más complejos. ${ }^{7-10}$

La implementación de estrategias interdisciplinarias de atención para el seguimiento de pacientes con AG demostró ser muy eficaz. ${ }^{6,11}$ A partir de 2008, el Servicio de Neumonología del Hospital Garrahan cuenta con un Programa de Atención de Niños con AG Problemática (PANAG) en el que participan neumonólogos, enfermeras, kinesiólogos y psicólogos, con una modalidad especial de atención con visitas frecuentes, administración gratuita de medicación, desarrollo de actividades educativas, entre otras. ${ }^{6}$

El objetivo de este estudio fue analizar las características clínicas, funcionales y terapéuticas de pacientes con AGRT y AGDC en seguimiento en un hospital público, bajo un protocolo especial de atención.

\section{MATERIAL Y MÉTODOS}

Se trata de un estudio transversal, observacional y analítico en el que se analizaron los resultados de la aplicación de un protocolo de atención en los pacientes seguidos en el PANAG. Los niños ingresaron al programa cuando no habían logrado controlar los síntomas con altas dosis de corticoides inhalados (CI) (asma problemática), asistían mensual o bimensualmente y, en caso de exacerbaciones, eran evaluados todos los días en el Servicio de
Neumonología o en Emergencias del Hospital. ${ }^{6}$

En este estudio efectuado entre junio y diciembre de 2016, se incluyeron todos los niños de ambos sexos, entre 6 y 18 años, en seguimiento en el programa con diagnóstico presuntivo de AG de acuerdo con la clasificación de la Iniciativa Global para el Asma: síntomas diarios, exacerbaciones frecuentes, síntomas nocturnos habituales, limitación de la actividad física y requerimiento de altas dosis de CI $(\geq 800 \mu \mathrm{g}$ de budesonida o equivalente) y broncodilatadores $\beta_{2}$ agonistas de acción prolongada (long-acting $\beta 2-$ agonists; LABA, por sus siglas en inglés) para mantener el control de la enfermedad. ${ }^{4}$ Se excluyeron los pacientes con fibrosis quística, bronquiolitis obliterante o displasia broncopulmonar.

Los pacientes fueron evaluados de manera interdisciplinaria según el protocolo de la OMS con el objetivo de diferenciar entre AGRT y AGDC, y fueron seguidos en el mismo programa luego de su participación. El protocolo incluía la reevaluación durante 6 meses de diagnóstico, grado de control del asma, factores asociados a la falta de control (comorbilidades, falta de adherencia al tratamiento, técnica inhalatoria incorrecta y factores ambientales) y ajuste del tratamiento ${ }^{6-9}$ (Figura 1). Estos aspectos se consideraron durante la evaluación, que incluyó 6 visitas.

Se definieron como AGDC los casos en los que, luego de la confirmación diagnóstica, se detectaron factores, tales como errores en la técnica y / o adherencia a la medicación según la evaluación del equipo tratante, dificultades en el acceso a la medicación o trastornos emocionales, y que, luego de su corrección, se pudo controlar la enfermedad. Se definió como AGRT cuando, luego de considerarse los factores modificables, no se logró un adecuado control del asma (síntomas diurnos $>2$ veces por semana, despertares nocturnos, salbutamol $>2$ veces por semana, limitación en la actividad física) y se requirieron altas dosis de tratamiento $(\mathrm{CI}, \mathrm{LABA}$, montelukast) y, eventualmente, omalizumab o corticoides orales para lograr un adecuado control. ${ }^{1,4,5}$

En ambos grupos, se evaluaron comorbilidades (rinitis, sinusitis, reflujo gastroesofágico, obesidad). La pHmetría esofágica o impedanciometría fue solicitada por el gastroenterólogo. Cuando se sospecharon diagnósticos alternativos (ejemplo: bronquiolitis obliterante) $\mathrm{u}$ otras enfermedades coexistentes 
(ejemplo: bronquiectasias), se realizaron tomografías axiales computadas de alta resolución (TACAR) de tórax y de senos paranasales, que fueron evaluadas por un radiólogo. En todos los casos, se efectuaron test del sudor, serología para virus de la inmunodeficiencia humana (VIH), dosaje de inmunoglobulinas y derivado proteico purificado (purified protein derivative; PPD, por sus siglas en inglés). Los niños con un índice de masa corporal igual al percentilo 95 o mayor fueron considerados obesos. ${ }^{12}$ Los pacientes con patologías asociadas fueron referidos a un especialista para control y tratamiento.

La adherencia y la técnica inhalatoria fueron consideradas en cada visita. La adherencia se evaluó de manera interdisciplinaria calculando el número total de dosis que debía recibir el paciente en el período determinado entre las consultas programadas y el número de dosis remanentes en el contador de dosis del inhalador.

Las recomendaciones sobre control medioambiental (tabaquismo domiciliario y contaminantes medioambientales de la vivienda) y técnica y adherencia al tratamiento fueron abordadas en talleres para padres y niños (actividades lúdicas, ejercicios físicos, entrenamiento kinésico de patrones respiratorios normales y técnicas de relajación para autocontrol) coordinados por psicólogos y kinesiólogos. ${ }^{6}$

La función pulmonar se evaluó en cada visita utilizando un espirómetro Medgraphics (MGC Diagnostics). Se efectuaron espirometrías, en las que se analizaron la capacidad vital forzada
(CVF), el volumen espiratorio forzado en el primer segundo $\left(\mathrm{VEF}_{1}\right)$, la relación $\mathrm{VEF}_{1} / \mathrm{CVF}$ y el flujo espiratorio forzado al $25 / 75 \%$ de la CVF (FEF 25/75\%) siguiendo los criterios de la American Thoracic Society/European Respiratory Society (ATS / ERS). ${ }^{13}$ Se consideró la espirometría inicial y a los 6 meses. Todos los parámetros se expresaron como porcentajes.

El control del asma se evaluó mediante el test del control del asma (TCA). Los valores $\geq 20$ indicaron asma controlado. ${ }^{14}$ En el estudio, se incluyó el resultado del TCA efectuado a los 6 meses de seguimiento.

Los objetivos del estudio fueron explicados a los pacientes, padres o tutores legales, quienes consintieron su participación.

\section{ESTADÍSTICA}

Se describió la distribución de los valores dentro de cada variable mediante medidas de tendencia central y dispersión o en forma categórica. Las variables categóricas se compararon con la prueba de chi². Las variables continuas se compararon mediante la prueba de Student o la prueba de Wilcoxon. Los valores de $\mathrm{p} \leq 0,05$ se consideraron significativos. Se utilizó el programa Stata XIII (Stata-Corp, College Station, TX).

\section{RESULTADOS}

Un total de 69 niños que se encontraban en seguimiento en el PANAG fueron incluidos en el estudio. El 45\% ( $\mathrm{n}=31)$ fueron varones; edad media de 12,4 años (desvío estándar-DE-4,1).

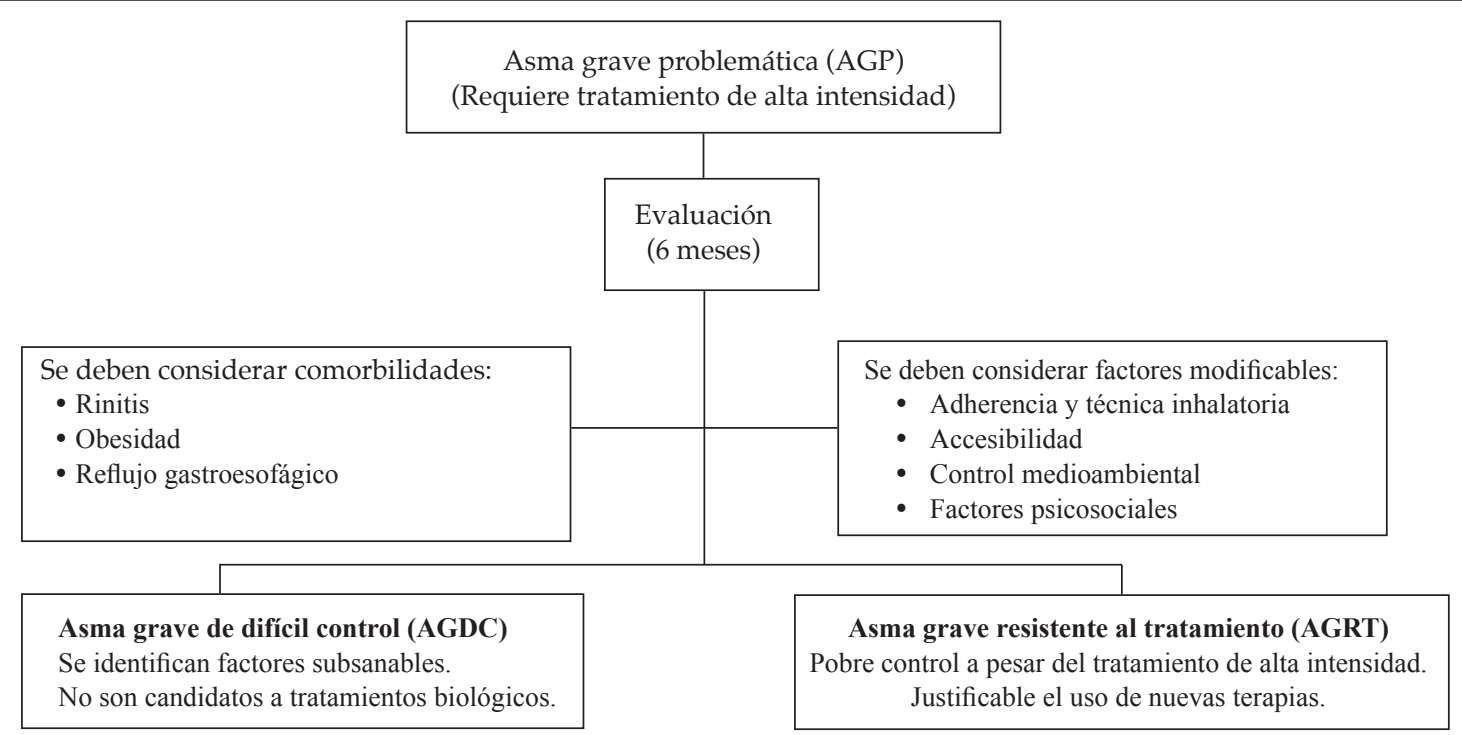


El 48\% (n=33) fue definido como AGDC y el 52\% $(\mathrm{n}=36)$, como AGRT. Las características de los pacientes se presentan en la Tabla 1.

Todos los niños con AGRT $(n=36)$ fueron hospitalizados por asma previo al inicio del seguimiento, mientras que el $87,8 \%(n=29)$ de los pacientes con AGDC requirieron internación $(p=0,03)$. El $63 \%$ de los casos con AGRT $(n=23)$ requirió la Unidad de Cuidados Intensivos (UCI), de los cuales el $82 \%(n=19)$ necesitó asistencia ventilatoria (AV) y un paciente fue traqueostomizado. En el grupo con AGDC, en cambio, el $54 \%(n=18)$ requirió la UCI y solo el $33 \%(\mathrm{n}=11)$ necesitó AV $(\mathrm{p}=0,03)$.

Las comorbilidades, inmunoglobulina $\mathrm{E}$
(IgE) y eosinofilia en sangre fueron similares en ambos grupos. Un mayor número de pacientes no cumplieron con el tratamiento en el grupo con $\operatorname{AGDC}(p=0,01)($ Tabla 1$)$.

La TACAR de tórax fue efectuada en 30 casos con AGRT (83,3\%), mientras que, en el grupo con AGDC, se solicitó en 6 pacientes (18\%) $(p=0,0001)$. Entre los hallazgos, se observaron atrapamiento aéreo, atelectasias laminares y engrosamiento peribronquial. En 2 pacientes con AGRT, se observaron bronquiectasias pequeñas en el lóbulo medio.

El grupo con AGRT presentó una incapacidad ventilatoria obstructiva leve, evidenciada únicamente por la disminución de la relación

TABla 1. Características de los niños con diagnóstico de asma grave de difícil control $(n=33)$ y asma grave resistente al tratamiento $(n=36)$

\begin{tabular}{|c|c|c|c|}
\hline Variables & $\begin{array}{c}\text { Asma grave de difícil control } \\
48 \%(n=33)\end{array}$ & $\begin{array}{l}\text { Asma grave resistente al tratamiento } \\
\qquad 52 \%(\mathrm{n}=36)\end{array}$ & Valor de $p$ \\
\hline Sexo (masculino) & $48,3 \%(\mathrm{n}=15)$ & $51,6 \%(n=16)$ & 0,5 \\
\hline Edad (años) & $13,7(12,2-15)^{*}$ & $11,2(10-12,5)^{*}$ & 0,01 \\
\hline Edad de inicio de los síntomas (meses) & $2,2(1,4-3)^{*}$ & $1(0,6-1,4)^{*}$ & 0,02 \\
\hline Historia familiar de asma & $52 \%(\mathrm{n}=15)$ & $48 \%(\mathrm{n}=14)$ & 0,6 \\
\hline $\operatorname{IgE}$ sérica $(\mathrm{UI} / \mathrm{ml})$ & $904(558-1249)^{*}$ & $1249(1045-1433)^{*}$ & 0,7 \\
\hline Eosinofilia & $452(332-572)^{*}$ & $522(338-706)^{*}$ & 0,5 \\
\hline Rinitis & $48 \%(\mathrm{n}=16)$ & $41,6 \%(n=15)$ & 0,6 \\
\hline Obesidad & $21 \%(\mathrm{n}=7)$ & $11 \%(\mathrm{n}=4)$ & 0,2 \\
\hline Reflujo gastroesofágico & $15 \%(\mathrm{n}=5)$ & $11 \%(\mathrm{n}=4)$ & 0,06 \\
\hline Fumadores domiciliarios & $39 \%(\mathrm{n}=13)$ & $33,3 \%(n=12)$ & 0,6 \\
\hline Sin adherencia al tratamiento & $86 \%(\mathrm{n}=28)$ & $56 \%(\mathrm{n}=20)$ & 0,01 \\
\hline
\end{tabular}

IgE: inmunoglobulina E.

${ }^{*}$ Los valores están expresados como promedio con intervalo de confianza (IC 95\%).

TABLA 2. Función pulmonar de niños con asma grave de difícil control ( $n=33$ ) y asma grave resistente al tratamiento ( $n=36)$ al inicio y a los 6 meses de seguimiento

\begin{tabular}{lccc}
\hline Variables & $\begin{array}{c}\text { Asma grave de difícil control } \\
\mathbf{4 8} \% \mathbf{( n = 3 3 )}\end{array}$ & $\begin{array}{c}\text { Asma grave resistente al tratamiento } \\
\mathbf{5 2 \%} \mathbf{( n = 3 6 )}\end{array}$ & Valor de $p$ \\
\hline CVF inicial & $94,7 \%(89,5-100)$ & $99 \%(93-105)$ & 0,2 \\
CVF final & $98,8 \%(93-103)$ & $105,7 \%(101-109,7)$ & 0,05 \\
Valor de $p$ & 0,04 & 0,01 & 0,5 \\
VEF $_{1}$ inicial & $89,7 \%(89-95)$ & $87 \%(80-94)$ & 0,1 \\
$\mathrm{VEF}_{1}$ final & $93,9 \%(89-98)$ & $98,4 \%(93-104)$ & 0,009 \\
Valor de $p$ & 0,04 & 0,0001 & 0,6 \\
$\mathrm{VEF}_{1} /$ CVF inicial & $85,5(82-88)$ & $79(75-83)$ & 0,3 \\
$\mathrm{VEF}_{1} /$ CVF final & $84(81-86)$ & $83(80-86)$ & 0,003 \\
Valor de $p$ & 0,2 & $74,5 \%(60-88)$ & 0,7 \\
FEF 25/75\% inicial & $81,8 \%(72-91)$ & $85,7 \%(75-96)$ & 0,02 \\
FEF 25/75\% final & $88,4 \%(76-99)$ & & \\
Valor de $p$ & 0,05 &
\end{tabular}

Los valores están expresados como promedios con intervalo de confianza (IC 95\%).

$\mathrm{CVF}$ : capacidad vital forzada; $\mathrm{VEF}_{1}$ : volumen espiratorio forzado en el primer segundo; $\mathrm{VEF}_{1} / \mathrm{CVF}$ : relación entre el volumen espiratorio forzado en el primer segundo y la capacidad vital forzada; FEF 25/75\%: flujo espiratorio forzado al 25/75\% de la capacidad vital forzada.

Para analizar los resultados entre los grupos, se aplicó el test de Student. Para analizar los datos al inicio y a los 6 meses dentro del mismo grupo, se utilizó el test de Student para datos apareados. 
$\mathrm{VEF}_{1} / \mathrm{CVF}$, a diferencia de aquellos con AGDC, en los que la espirometría basal fue normal $(p=0,009)$. A los 6 meses, se evidenció un incremento significativo en todos los parámetros en ambos grupos (Tabla 2).

A los 6 meses de seguimiento, el grupo con AGRT permaneció con dosis de CI y LABA significativamente mayores que los casos con $\operatorname{AGDC}(p=0,0001$ y $p=0,006)$. Mayor número de pacientes requirieron montelukast en el grupo con AGRT y omalizumab fue únicamente indicado en este grupo ( $\mathrm{p}=0,05$ y $\mathrm{p}=0,0001)$ (Tabla 3$)$. A los 6 meses de seguimiento, $75 \%(\mathrm{n}=25)$ del grupo con AGDC y $80 \%(\mathrm{n}=25)$ del grupo con AGRT tuvieron adecuado control del asma $(p=0,6)$.

\section{DISCUSIÓN}

En este estudio, se presentaron las características de una cohorte de pacientes con diagnóstico de AG problemática en seguimiento bajo un protocolo especial de atención, en un hospital público. Luego de 6 meses de evaluación, más del $50 \%$ de los casos se definieron como AGRT. En el resto de los pacientes, las intervenciones en los factores modificables permitieron su recategorización como AGDC ajustando los tratamientos oportunamente.

La diferenciación entre el grupo con AGRT, en el que los síntomas permanecen no controlados a pesar de recibir el esquema terapéutico más alto recomendado o en el que el adecuado control solo puede mantenerse con dosis altas, del grupo con AGDC, en el que el asma puede controlarse después de eliminar los factores reversibles, constituye un desafío. ${ }^{8,9}$ Se han publicado pocos estudios que evalúen estas poblaciones. ${ }^{15,16}$

Con este objetivo, la OMS propuso un protocolo para facilitar el seguimiento de pacientes con AG no controlada. ${ }^{7,8}$ Inicialmente, define el diagnóstico como AG problemática. ${ }^{7,8}$ Estos niños presentan síntomas persistentes y/o exacerbaciones graves, a pesar de recibir altas dosis de CI. ${ }^{8-10}$ Requieren una evaluación sistemática: confirmar el diagnóstico, evaluar cuidadosamente la adherencia al tratamiento y la técnica inhalatoria, el adecuado manejo de comorbilidades y el control del medioambiente. Luego de este abordaje, el 50-75\% de estos niños presentarán AGDC por causas potencialmente modificables, como las antes mencionadas. La evolución es favorable con los tratamientos disponibles. El 25-50\% restante está constituido por AGRT, la forma más grave de la enfermedad, en la que se consideran tratamientos más específicos. Ambos casos requieren supervisión regular. ${ }^{16,17}$

En este estudio, en cada consulta, el equipo tratante evaluó sistemáticamente la técnica inhalatoria y trabajó sobre la adherencia y el control del medioambiente y la vivienda del niño. Estas intervenciones fueron individuales y grupales a través de talleres para padres y niños. ${ }^{6}$

Se observaron diferencias entre los grupos. Los niños con AGRT iniciaron los síntomas precozmente en la vida, en concordancia con los datos publicados. ${ }^{18}$ Todos los casos habían sido hospitalizados por asma y más de la mitad requirió la $\mathrm{UCI}$ y / o $\mathrm{AV}$, mientras que un número significativamente menor, aunque, de igual manera, relevante, se había hospitalizado en el grupo con AGDC, lo que puso de manifiesto la gravedad del asma en ambos grupos.

En esta serie, como en otras, los niveles basales de IgE o la eosinofilia no fueron parámetros que diferenciaran los grupos. ${ }^{15,16}$ La frecuencia de reflujo gastroesofágico, rinitis u obesidad, tanto como de tabaquistas domiciliarios, fue similar a otras series publicadas y no hubo diferencias entre los grupos. ${ }^{15}$

La función pulmonar de pacientes adultos con AG está habitualmente afectada. En pediatría, aun en los casos con AGRT, la espirometría puede

TABLA 3. Tratamiento administrado a pacientes con diagnóstico de asma grave de difícil control ( $n=33$ ) y asma grave resistente al tratamiento $(n=36)$ luego de 6 meses del seguimiento

\begin{tabular}{|c|c|c|c|}
\hline Variables & $\begin{array}{l}\text { Asma grave de difícil control } \\
\qquad 48 \%(n=33)\end{array}$ & $\begin{array}{l}\text { Asma grave resistente al tratamiento } \\
\qquad 52 \%(n=36)\end{array}$ & Valor de $p$ \\
\hline Corticoides inhalados ( $\mu$ g/día) & $601(509-693)^{*}$ & $836(762-910)^{*}$ & 0,0001 \\
\hline LABA $(\mu g /$ día $)$ & $56(46-67,3)^{*}$ & $77(66,8-87,4)^{*}$ & 0,006 \\
\hline Montelukast & $12 \%(\mathrm{n}=4)$ & $30 \%(\mathrm{n}=11)$ & 0,05 \\
\hline Meprednisona oral & $0 \%(\mathrm{n}=0)$ & $5,5 \%(\mathrm{n}=2)$ & 0,1 \\
\hline Omalizumab & $0(\mathrm{n}=0)$ & $41,6 \%(\mathrm{n}=15)$ & 0,0001 \\
\hline
\end{tabular}

* Los valores están expresados en promedio con intervalo de confianza (IC 95\%).

LABA: $\beta_{2}$ agonistas adrenérgicos de acción prolongada. 
ser normal o evidenciar una obstrucción leve..$^{18-20}$ Algunos autores, sin embargo, han sugerido que la función pulmonar en pediatría permitiría discriminar entre AGRT y AGDC, aunque otros sostienen lo contrario. ${ }^{15,16,21}$ En este estudio, únicamente los niños con AGRT presentaron una obstrucción leve pero significativa al inicio del seguimiento, evidenciada por una reducción de la $V_{E F} / C V F$, que luego se normalizó. A los 6 meses, ambos grupos incrementaron todos los parámetros espirométricos de manera significativa. La optimización del tratamiento generó una mejoría de la función pulmonar independientemente de los valores basales normales o casi normales en ambos grupos, lo que puso de manifiesto la falta de sensibilidad del método para detectar el grado de compromiso inflamatorio de la vía aérea. Es llamativo que pacientes con una espirometría basal casi normal permanezcan sin adecuado control de la enfermedad. Una hipótesis que podría explicar los cambios leves observados en la relación $\mathrm{VEF}_{1}$ / CVF en pacientes con síntomas frecuentes estaría dada por el aumento del tono broncomotor y por su labilidad. De todos modos, no se puede descartar que cambios histopatológicos, como la remodelación bronquial, ya estén presentes en escolares y adolescentes. ${ }^{18,22,23}$

La adecuada categorización como AGDC o AGRT permite no solo minimizar la solicitud de estudios y procedimientos innecesarios, sino también disminuir los riesgos de efectos adversos de la medicación. ${ }^{16}$ El control del asma es posible en AGDC con dosis menores de tratamiento y menos fármacos. En AGRT, sin embargo, puede no alcanzarse un adecuado control del asma, incluso cuando se eliminan factores potenciales de riesgo. Estos casos requieren dosis de CI más altas y son candidatos a nuevas estrategias terapéuticas, como los anticuerpos monoclonales (omalizumab) o los corticoides orales continuos..$^{8,9,17,24}$ En esta serie, la categorización de los pacientes permitió personalizar los tratamientos. Un alto porcentaje de niños con AGDC lograron controlar los síntomas con dosis significativamente menores de CI/LABA y menos pacientes requirieron montelukast, comparados con el grupo con AGRT. Únicamente en este grupo, hubo pacientes tratados con omalizumab o corticoides orales.

Los costos del AG pueden comprometer hasta un $29 \%$ del ingreso familiar ${ }^{15}$ En este estudio, se garantizó su accesibilidad, ya que la medicación y las aerocámaras fueron provistas en forma gratuita por el Hospital para controlar una causa importante de falla del tratamiento, especialmente, en una población con recursos económicos limitados. ${ }^{25}$

Otro factor importante asociado con la falta de control es la mala adherencia al tratamiento. Existen múltiples razones para no cumplir con los tratamientos, que incluyen costos, regímenes de tratamiento complejos, riesgo de efectos secundarios y falta de supervisión de los padres, entre otros. ${ }^{25,26} \mathrm{~A}$ pesar de que no existen métodos completamente confiables para verificarla, se recomienda controlar y promover el cumplimiento de las indicaciones terapéuticas con los métodos disponibles al momento de la consulta. Una adherencia satisfactoria se define como la administración de, al menos, el $80 \%$ de las dosis prescritas. Sin embargo, solo el $24 \%$ de los pacientes con AGDC tienen adherencia al tratamiento $\geq 80 \%$, mientras que el $41 \%$ de los niños con AGRT cumplen los tratamientos indicados. ${ }^{16}$ En el presente estudio, un alto porcentaje de pacientes con AGDC no cumplió con los tratamientos indicados. De todos modos, considerando que, habitualmente, la adherencia reportada por los padres es mayor que la verdadera, la falta de cumplimiento del tratamiento en ambos grupos fue alarmante, aunque concordante con otras publicaciones. ${ }^{16}$

El AG constituye una patología de muy baja prevalencia (5\% del total de asmáticos). En esta serie, fue analizado un elevado número de casos con AG, seguidos de modo adecuado en un programa especial de atención, lo que facilitó la recolección de datos. Sin embargo, el carácter observacional de este estudio no excluyó completamente potenciales confundidores.

\section{CONCLUSIÓN}

En esta población, el inicio precoz de los síntomas y el antecedente de internaciones en la UCI con requerimientos de AV fueron características significativamente presentes en pacientes con AGRT comparados con los casos con AGDC. Asimismo, la aplicación de un protocolo de atención permitió determinar que la falta de adherencia al tratamiento fue el factor modificable significativamente más observado en el grupo con AGDC, lo que posibilitó reducir las dosis de CI de manera significativa en estos niños. Un alto porcentaje de casos en ambos grupos logró controlar la enfermedad, lo que confirmó la relevancia de este abordaje. 


\section{REFERENCIAS}

1. Comité Nacional de Neumonología; Comité Nacional de Alergia; Comité Nacional de Medicina Interna; et al. Guía de diagnostico y tratamiento: asma bronquial en niños $\geq 6$ años. Actualización 2016. Resumen ejecutivo. Arch Argent Pediatr 2016;114(6):595-6.

2. Mallol J, Crane J, von Mutius E, et al. The International Study of Asthma and Allergies in Childhood (ISAAC) Phase Three: a global synthesis. Allergol Immunopathol (Madr) 2013;41(2):73-85.

3. Argentina. Ministerio de Salud. Secretaria de Políticas, Regulación e Institutos. Dirección de Estadísticas e Información de Salud. Estadísticas Vitales: Información básica año2013. [Acceso:8 deseptiembrede2017]. Disponible en: http: / / www.bvs.org.ar/pdf/ anuario13.pdf.

4. Global Strategy for Asthma Management and Prevention. Global Initiative for Asthma (GINA) 2016. [Acceso: 8 de septiembre de 2017]. Disponible en: http://ginasthma. org / wp-content/ uploads / 2016 / 04 / GINA-2016-mainreport_tracked.pdf.

5. British guideline on the management of asthma. A national clinical guideline October 2014;141. British Thoracic Society. [Acceso: 8 de septiembre de 2017]. Disponible en: https: / / www.brit-thoracic.org.uk/document-library/clinicalinformation/asthma/btssign-asthma-guideline-2014/.

6. Giubergia V, Fridman N, González Pena H. Evaluación del impacto de un programa de atención de niños con asma grave. Arch Argent Pediatr 2012;110(5):382-7.

7. Bush A, Zar HJ.WHO universal definition of severe asthma. Curr Opin Allergy Clin Immunol 2011;11(2):115-21.

8. Bush A, Hedlin G, de Benedictis F, et al. Severe childhood asthma: a common international approach? Lancet 2008;372(9643);1019-21.

9. Hedlin G, Bush A, Lødrup Carlsen K, et al. Problematic severe asthma in children, not one problem but many: a GA2LEN initiative. Eur Respir J 2010;36(1):196-201.

10. Bush A, PedersenS, Hedlin G, etal. Pharmacological treatment of severe, therapy-resistant asthma in children: what can we learn from where? Eur Respir J 2011;38(4):947-58.

11. Brandão HV, Cruz CMS, Santos Ida S Jr, et al. Hospitalizations for asthma: impact of a program for the control of asthma and allergic rhinitis in Feira de Santana, Brazil. J Bras Pneumol 2009;35(8):723-9.

12. Centers for Diseases Control and Prevention. National Center for Health Statistics. National Health and Nutrition Examination Survey. Anthropometric Reference Data,
United States 1988-1994. [Acceso: 8 de septiembre de 2017]. Disponibleen: http: / / www.cdc.gov/nchs/about/major / nhanes / Anthropometric\%20Measures.htm.

13. Miller MR, Hankinson J, Brusasco V, et al. Standardization of spirometry. Eur Respir J 2005;26(2):319-38.

14. Rodrigo GJ, Árcos JP, Nannini LJ, et al. Reliability and factor analysis of the Spanish version of the asthma control test. Ann Allergy Asthma Immunol 2008;100(1):17-22.

15. De Andrade WC, Lasmar LM, RicciCde A, et al. Phenotypes of severe asthma among children and adolescents in Brazil: a prospective study. BMC Pulm Med 2015;15:36.

16. Cook J, Beresford F, Fainardi V, et al. Managing the pediatric patient with refractory asthma: a multidisciplinary approach. J Asthma Allergy 2017;10:123-30.

17. Martin Alonso A, Saglani S. Mechanisms Mediating Pediatric Severe Asthma and Potential Novel Therapies. Front Pediatr 2017;5:154.

18. Rodrigues AM, Roncada C, Santos G, et al. Clinical characteristics of children and adolescents with severe therapy-resistant asthma in Brazil. J Bras Pneumol 2015;41(4):343-50.

19. Jenkins HA, Cherniack R, Szefler SJ, et al. A comparison of the clinical characteristics of children and adults with severe asthma. Chest 2003;124(4):1318-24.

20. Lang AM, Konradsen J, Carlsen $\mathrm{KH}$, et al. Identifying problematic severe asthma in the individual child - does lung function matter? Acta Paediatr 2010;99(3):404-10.

21. Konradsen JR, Nordlund B, Lidegran M, et al. Problematic severe asthma: a proposed approach to identifying children who are severely resistant to therapy. Pediatr Allergy Immunol. 2011;22(1 Pt 1):9-18.

22. Saglani S, Payne DN, Zhu J, et al. Early detection of airway wall remodeling and eosinophilicinflammationin preschool wheezers. Am J Respir Crit Care Med 2007;176(9):858-64.

23. Payne DN, Rogers AV, Adelroth E, et al. Early thickening of the reticular basement membrane in children with difficult asthma. Am J Respir Crit Care Med 2003;167(1):78-82.

24. Neffen H, Vidaurreta S, Balanzat A, et al. Asma de difícil control en niños y adolescentes: estrategias diagnósticoterapéuticas. Medicina (B Aires) 2012;72(5):403-13.

25. Anderson W, Szefler S. New and future strategies to improve asthma control in children. J Allergy Clin Immunol 2015;136(4):848-59.

26. Bourdin A, Halimi L, Vachier I, et al. Adherence in severe asthma. Clin Exp Allergy 2012;42(11):1566-74. 